\title{
Study of the Jet-Induced Mixing of Two-Layer Density-Stratified Fluid in a Rectangular Tank
}

\author{
Shogo Shakouchi ${ }^{1,2}$, Shota Fukue ${ }^{2}$ and Tomomi Uchiyama ${ }^{3}$ \\ 1. Development Section, Chubu Electric Power Co., Inc., Nagoya 461-8680, Japan \\ 2. Graduate School of Information Science, Nagoya University, Nagoya 464-8601, Japan \\ 3. EcoTopia Science Institute, Nagoya University, Nagoya 464-8603, Japan
}

Received: August 26, 2014 / Accepted: October 15, 2014 / Published: February 28, 2015.

\begin{abstract}
The mixing phenomena of a two-layer density-stratified fluid induced by a jet in a tank are experimentally investigated. The upper and lower fluids are water and a NaCl-water solution, respectively, with the lower fluid issued vertically upward from a nozzle at the bottom of the tank. The jet Reynolds number Re, defined by the jet velocity and the water kinematic viscosity, ranges from 90 to 4,200. The mass concentration of the $\mathrm{NaCl}$-water solution $C_{0}$ is less than 0.08 . The flow visualization makes clear the jet behavior relative to the density interface between the upper and lower fluids. The measurement of the concentration distribution of the water paint issued with the jet highlights the effects of $R e$ and $C_{0}$ on the mixing between the jet and the ambient fluid. The measurement of the fluid velocity distribution with a PIV (particle image velocimetry) system successfully elucidates the relationship between the velocity field and the resultant mixing.
\end{abstract}

Key words: Jet, density-stratified fluid, mixing, intrusion, concentration, PIV.

\section{Introduction}

In a storage tank of LNG (liquefied natural gas), the density stratification of the LNG is produced when another LNG is loaded on top of the LNG. This is because the density and components of LNG are specific to the producing areas and the purification plants. The density-stratified LNG becomes unstable over time because of temperature and composition variations. When density of the lower LNG becomes smaller than that of the upper one, a sudden mixing, known as rollover, occurs. Rollover generates large amounts of vaporized gases, causing severe damages to the storage tank. Therefore, the prevention and elimination of stratification are essential for the operation of LNG storage tanks.

Mixing phenomena of density-stratified fluid induced by jets in tank have thus far been studied with

Corresponding author: Tomomi Uchiyama, doctor of engineering, professor, research fields: fluid engineering and computational. E-mail: uchiyama@is.nagoya-u.ac.jp. laboratory-based experiments [1-6]. Mixing LNG by a jet issued from a nozzle mounted on the tank wall is considered to a promising technique to prevent and eliminate stratification in LNG storage tanks. The current authors [7] performed a laboratory-based experiment of two-layer density-stratified fluid in a rectangular tank. The upper and lower fluids were water and a NaCl-water solution, respectively, and the lower fluid was issued vertically upward from a nozzle on the tank bottom. The authors investigated the jet behavior by the flow visualization and clarified the effects of the jet Reynolds number, the concentration of the lower fluid and the fluid thickness. They also demonstrated that the maximum height of the jet can be predicted by the Froude number defined by the jet velocity and the density difference between the upper and lower fluids. The current authors [8] also proposed a simulation method for a jet issued into a two-layer density-stratified fluid. The method was based on a vortex in cell method, which discretizes the vorticity 
field into vortex elements and computes the time evolution of the flow by tracing the convection of each vortex element. Shakouchi et al. [8] performed the jet simulation at the same conditions as their previous experiment [7] and confirmed the validity of the simulation method by comparing the simulated results with the experimental ones. The abovementioned authors' studies [7,8] successfully clarified the jet behavior and presented the simulation method. But the mixing between the jet and the ambient fluid as well as the fluid velocity deeply interacting with the mixing is not fully elucidated.

In this study, the concentration distribution of the water paint issued with the jet is measured on the flow field investigated by the authors' previous work [7]. The distribution of the fluid velocity is also measured. On the basis of the measurements, the effects of the jet Reynolds number and the concentration of the lower fluid on the mixing between the jet and the ambient fluid are clarified. The knowledge of the velocity distribution, which contributes to the understanding of the jet-induced mixing, is also acquired.

\section{Experimental}

\subsection{Experimental Setup}

The experimental setup is the same as that used in the previous study of Shakouchi et al. [7]. The outline is shown in Fig. 1. Initially $t$ (at time) $=0$, a two-layer density - stratified fluid is in a static condition in a rectangular tank $\left(600 \times 200 \times 250 \mathrm{~mm}^{3}\right)$. The upper and lower fluids are water and a $\mathrm{NaCl}$-water solution, respectively. The tank is made of transparent acrylic resin to enable flow visualization. The vertical thicknesses of the upper and lower fluids are $z_{1}$ and $z_{2}$, respectively. The mass concentration of the $\mathrm{NaCl}$-water solution is denoted by $C_{0}$.

A nozzle with a circular cross-section with diameter $d$ of $10 \mathrm{~mm}$ and length 10 times $d$ is mounted at the center of the tank bottom. The nozzle outlet is positioned $20 \mathrm{~mm}$ above the tank bottom. The origin of the coordinates is set at the center of the nozzle outlet with the $x-y$ plane horizontal and the $z$-axis vertical. The nozzle centerline coincides with the z-axis. The nozzle is connected with a circular hole in the tank wall near the bottom via a tube, and a pump and flowmeter are installed between the hole and the nozzle.

\subsection{Experimental Method and Conditions}

At time $t \geq 0$, the lower fluid, the $\mathrm{NaCl}$-water solution, is issued vertically upward from the nozzle. The mean velocity at the nozzle outlet section is denoted by $U_{0}$. The fluid volume in the tank is maintained constant by using pump-driven fluid circulation.

To visualize the jet behavior, a small amount of fluorescent paint (Rhodamine B) is added to the jet.

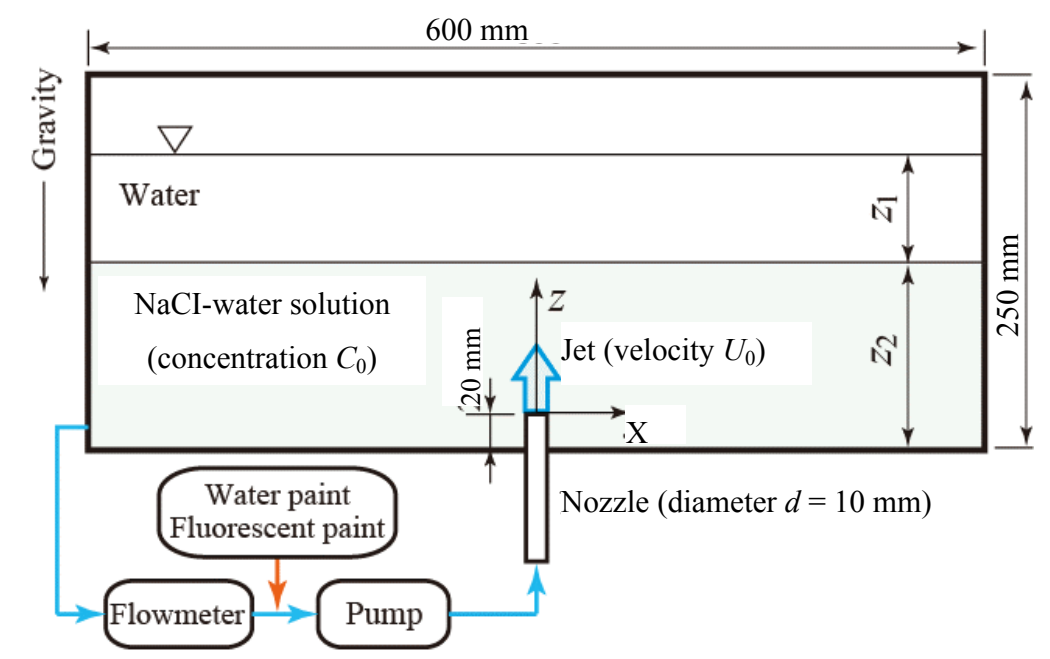

Fig. 1 Experimental setup. 
Laser light sheet

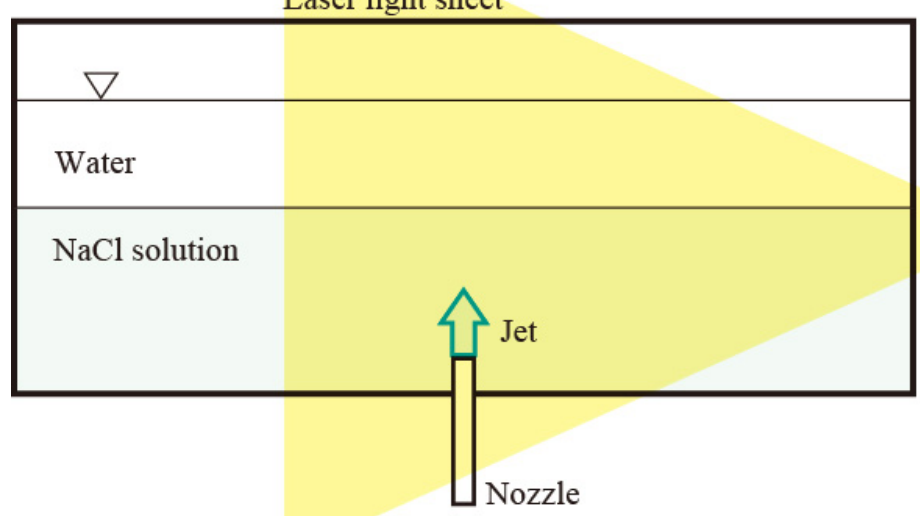

Laser light sheet

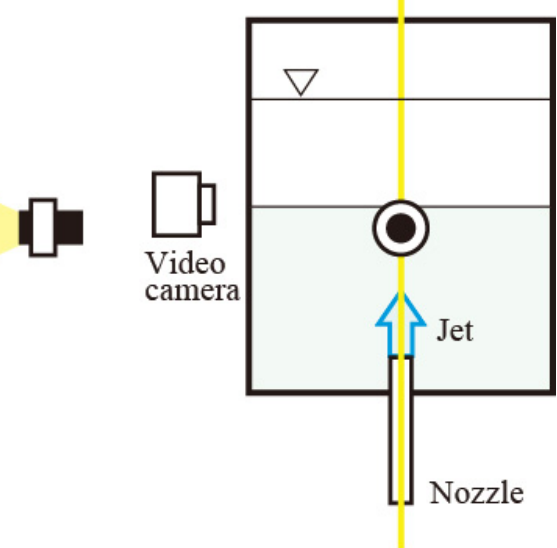

Fig. 2 Visualization of the central vertical cross-section of the flow field.

The images in the vertical plane passing through the nozzle centerline are captured by a video camera using a laser light sheet (power: $1 \mathrm{~W}$, wavelength: $532 \mathrm{~nm}$, thickness: $2 \mathrm{~mm}$ ), as shown in Fig. 2. The spatial resolution, framerate, and shutter speed of the camera are $640 \times 480$ pixels, $200 \mathrm{fps}$, and $1 / 200 \mathrm{~s}$, respectively.

To reveal the mixing phenomena, a small amount of white water paint is added to the jet, and the images in the vertical plane are captured by a flow visualization camera. The brightness of the water paint in the image is assumed to be proportional to the concentration $\Gamma$ of the paint. In order to normalize the $\Gamma$-value, $\Gamma$ is set to 1 at the nozzle exit and 0 in regions furthest from the nozzle. This is because the brightness, which is at the maximum at the nozzle exit, decreases with increasing distance from the nozzle.

The fluid velocity is measured by a PIV system. Nylon particles (mean diameter: $20 \mu \mathrm{m}$, specific weight: 1.02) are used as tracers. The particles images are acquired by using the laser light sheet and video camera used for the flow visualization.

The jet Reynolds number, $R e$, is defined by $U_{0} / v$, where $v$ is the kinematic viscosity of water. The non-dimensional time $t^{*}$ is defined by $t U_{0} / d$. Table 1 summarizes the experimental conditions.
Table 1 Experimental conditions.

\begin{tabular}{ll}
\hline Upper fluid & Water at 298 K \\
\hline Lower fluid & NaCl-water solution at $298 \mathrm{~K}$ \\
Nozzle inner diameter: $d$ & $10 \mathrm{~mm}$ \\
Size of rectangular tank & $600 \times 200 \times 250 \mathrm{~mm}^{3}$ \\
Reynolds number: $R e=d U_{0} / v$ & $90 \leq R e \leq 4,200$ \\
Concentration of NaCl-water & $C_{0} \leq 0.08$ \\
solution: $C_{0}$ & $20 \leq z_{1} \leq 60 \mathrm{~mm}$ \\
Thickness of upper fluid: $z_{1}$ & $20 \leq z_{2} \leq 80 \mathrm{~mm}$ \\
Thickness of lower fluid: $z_{2}$ &
\end{tabular}

\section{Results and Discussions}

\subsection{Jet Behavior Relative to Density Interface}

The jet behavior relative to the density interface between the upper and lower fluids can be classified into three patterns $\mathrm{A}, \mathrm{B}$, and $\mathrm{C}$ according to the Reynolds number Re and mass concentration of the NaCl-water solution $C_{0}$ [7]. Fig. 3 shows typical examples for $C_{0}=0.02$, where $z_{1}=40 \mathrm{~mm}$ and $z_{2}=70$ $\mathrm{mm}$. The images are acquired at $t^{*}=14$. The white area representing the fluorescent paint issued from the nozzle reveals the jet. In Pattern A, observed at $R e=95$ (Fig. 3a), the jet reaches the density interface without penetrating it. The top of the jet spreads almost horizontally outward beneath the interface and thus causes minimal mixing. In Pattern $\mathrm{B}$, observed at $R e=$ 476 , the jet penetrates the density interface and the non-axisymmetric flow structure becomes pronounced, 


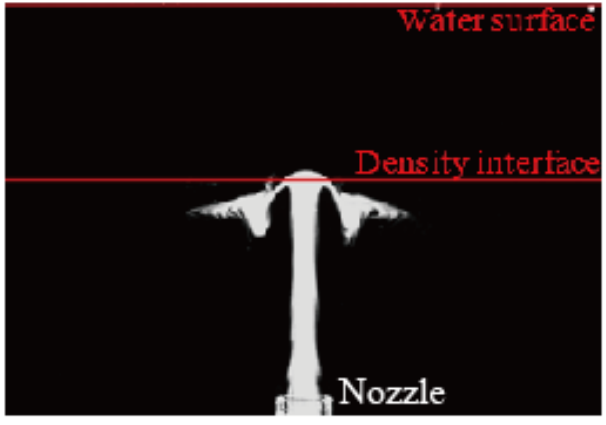

(a) Pattern $\mathrm{A}(R e=95)$

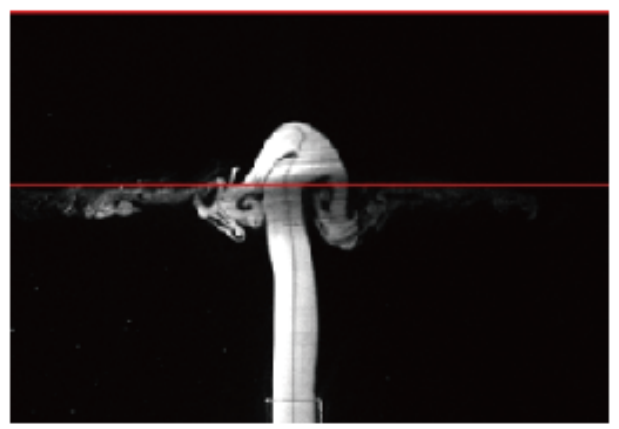

(b) Pattern B $(R e=476)$

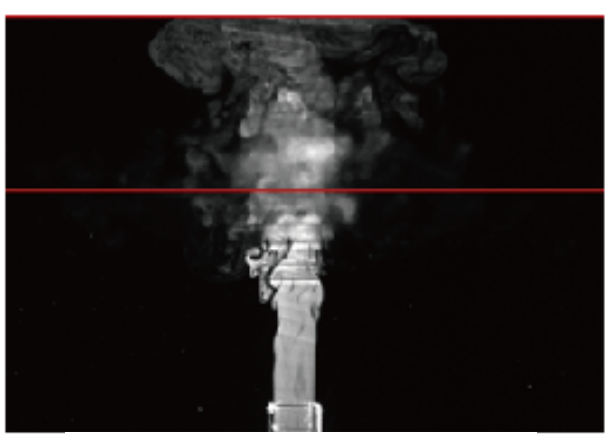

(c) Pattern C $(R e=2,378)$

Fig. 3 Jet behavior relative to the density interface at $t^{*}=$ $14\left(C_{0}=0.02, z_{1}=40 \mathrm{~mm}, z_{2}=70 \mathrm{~mm}\right)$.

as shown in Fig. 3b. The top of the jet falls back to the interface due to the gravitational effect, as the jet density is greater than the upper fluid density. Thus, the jet does not reach the upper boundary, i.e., the water surface. The fluid descending from the top of the jet spreads horizontally when it again reaches the density interface, which causes the interface to heave. The mixing layer is evident in a region along the interface. In Pattern $\mathrm{C}$, observed at $R e=2,378$ (Fig. 3c), the jet penetrating the density interface reaches the upper boundary and spreads significantly in the horizontal direction along the boundary. The horizontal flow along the density interface is also observed, with active mixing between the jet and the upper fluid.

Fig. 4 shows the pattern map of the jet behavior in the case of $z_{1}=40 \mathrm{~mm}$ and $z_{2}=70 \mathrm{~mm}$. Pattern A appears only when $R e$ is extremely low. The transition from Pattern $\mathrm{A}$ to $\mathrm{B}$ is not sensitive to $C_{0}$ and vice versa. The $R e$ range at which Pattern $\mathrm{B}$ appears broadens with increasing $C_{0}$. Note that an intermediate pattern exists between Patterns B and $\mathrm{C}$ when $C_{0} \geq 0.02$, as indicated by circular open symbols. The jet does not always reach the upper water surface but intermittently touches the surface.

When a fluid with density $\rho$ reaches the density interface from the outside, it flows in a horizontal direction along the interface if the condition of $\rho_{1}<\rho<$ $\rho_{2}$ is satisfied, where $\rho_{1}$ and $\rho_{2}$ are the densities of the upper and lower fluids, respectively [9]. Such flow is recognized as an intrusion of an internal density current. An example of this type of flow can be observed in the temperature-stratified water of a dam reservoir when muddy water mixed with sands flows into the reservoir and reaches the density interface. The horizontal flow along the density interface visualized in Fig. 3 corresponds to the intrusion of an internal density current.

\subsection{Distribution of Concentration of Water Paint Issued with Jet}

The concentration $\Gamma$ of the water paint issued with the jet from the nozzle is measured. Fig. 5 shows the change in $\Gamma$ along the vertical lines at $x / d=1,3$, and 5 . The measured conditions are the same as those of Fig. 3. The change at $R e=95$ is shown in Fig. 5a. The jet behavior is categorized as Pattern A. The jet reaches the density interface without penetrating it, as seen in Fig. 3a. Maximum $\Gamma$ occurs slightly below the interface $(z / d=5)$, and is the largest near the jet centerline $(x / d=$ $1)$, yet lower at the locations of $x / d=3$ and 5 far from the centerline. The effect of the jet appears only around the top of the jet near the density interface. Fig. $5 \mathrm{~b}$ 


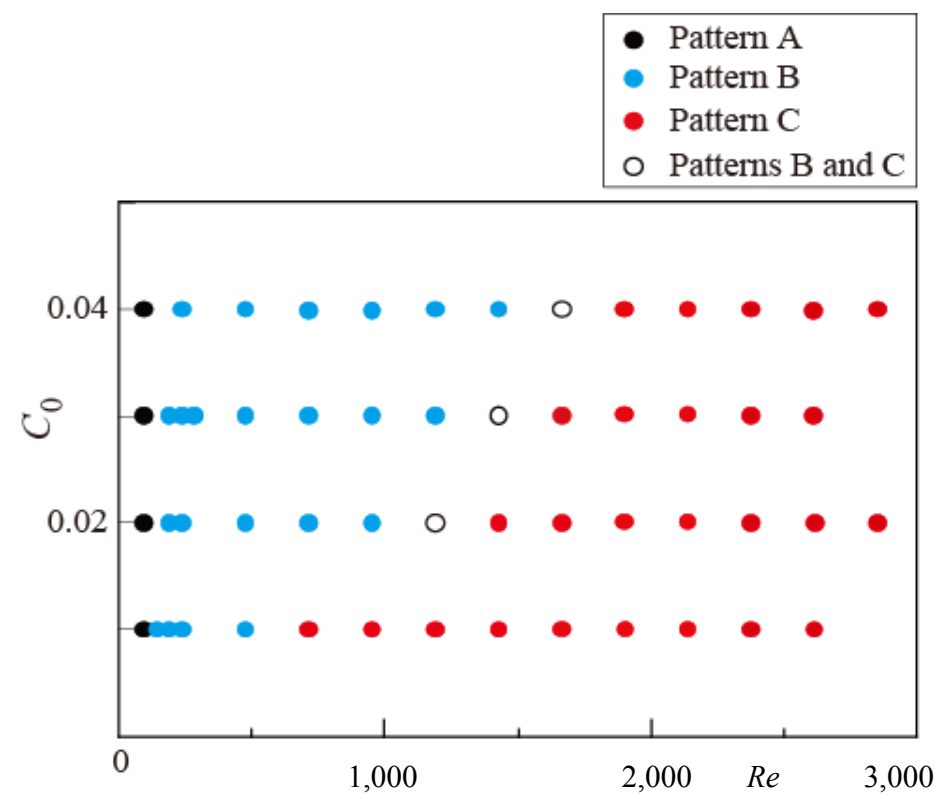

Fig. 4 Pattern map of jet behavior $\left(z_{1}=40 \mathrm{~mm}, z_{2}=70 \mathrm{~mm}\right)$.

shows the change in $\Gamma$ at $R e=476$. The jet behavior is categorized as Pattern B. The top of the jet penetrating the density interface falls back to the interface and spreads horizontally along the interface, as seen in Fig. $3 \mathrm{~b}$. At $x / d=1, \Gamma$ reaches the maximum on the interface, and the maximum value is larger than that at $R e=95$. One can confirm the mixing along the interface. The change at $R e=2,378$ is shown in Fig. $5 \mathrm{c}$. The jet behavior is categorized as Pattern $\mathrm{C}$. The jet penetrating the density interface reaches the upper water surface, as seen in Fig. $3 \mathrm{c}$. The $\Gamma$ value near the jet centerline $(x / d=1)$ is high in the broader region above the density interface as well as beneath the upper water surface, even at $x / d=5$. This is a product of the fluid flow along the water surface and thus reconfirms active mixing between the jet and the ambient fluid.

The flow along the density interface or the intrusion is reconfirmed by the fact that $\Gamma$ is at the maximum near the interface in every flow pattern, as shown in Fig. 5.

To investigate the time variation of $\Gamma$ on the density interface of the flow fields shown in Fig. 3, the variation is measured at two points $a$ and $b$ on either side of the jet centerline $(x=0)$. The distance between the points is the nozzle diameter $d$. The points $\mathrm{a}$ and $\mathrm{b}$ are superimposed on the flow image at $R e=476$, as shown in Fig. 6.

Fig. 7 shows the time variation of $\Gamma$ measured at points a and $\mathrm{b}$. The variations at $R e=95$ are shown in Fig. 7a. The measuring points are nearly located on the most leading end of the jet. Each value measured at points $\mathrm{a}$ and $\mathrm{b}$ fluctuates with a constant period. The phase difference between the measurements is very small. The jet is found to be almost axisymmetric. The mixing between the jet and the ambient fluid occurs extremely locally. When $R e=476, \Gamma$ also oscillates with a constant period as shown in Fig. 7b. The measuring points are located slightly below the top of the jet. The oscillating amplitude and period are much larger than those at $R e=95$, and the phase inversion occurs. These demonstrate that the top of the jet swings markedly in the horizontal direction on the density interface and that the mixing is activated there. The variations at $R e=2,378$ are shown in Fig. 7c. The measuring points are located near the centerline of the fully developed jet. The $\Gamma$-value is larger and fluctuates irregularly. There is no correlation between the measurements at points $\mathrm{a}$ and $\mathrm{b}$. One can find that eddies having various scales pass through the measuring points and that the mixing becomes more complicated and activated. 


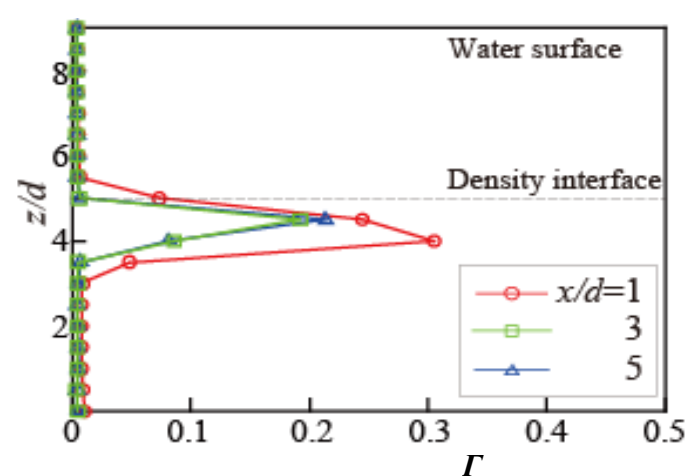

(a) $R e=95($ Pattern A)

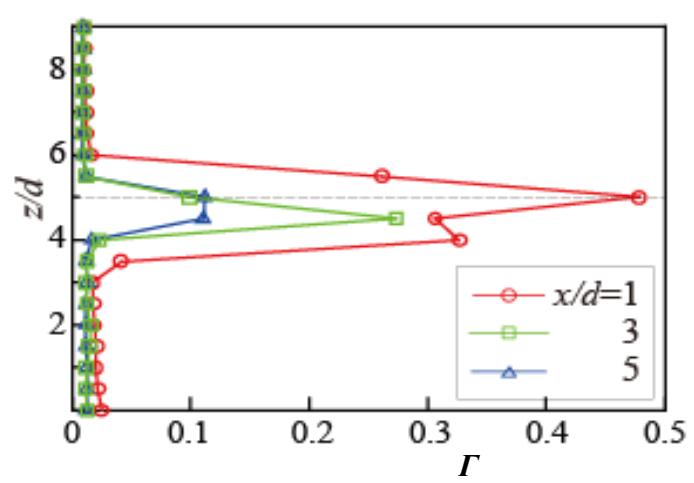

(b) $R e=476$ (Pattern $\mathrm{B})$

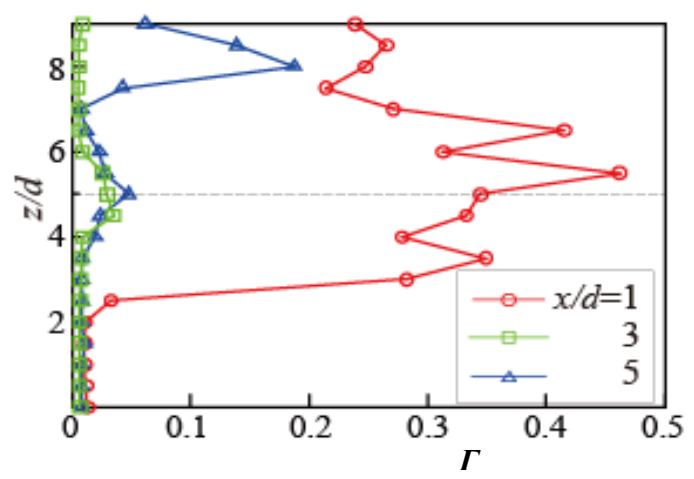

(c) $R e=2,378$ (Pattern $\mathrm{C}$ )

Fig. 5 Vertical distribution of $\Gamma$ at $t^{*}=14\left(C_{0}=0.02, z_{1}=40\right.$ $\mathrm{mm}, z=70 \mathrm{~mm})$.

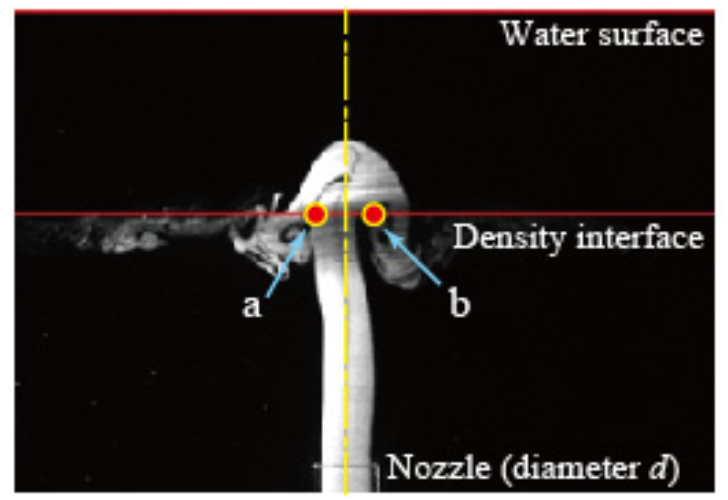

Fig. 6 Measuring points a and $b$ for $\Gamma$ on density interface.

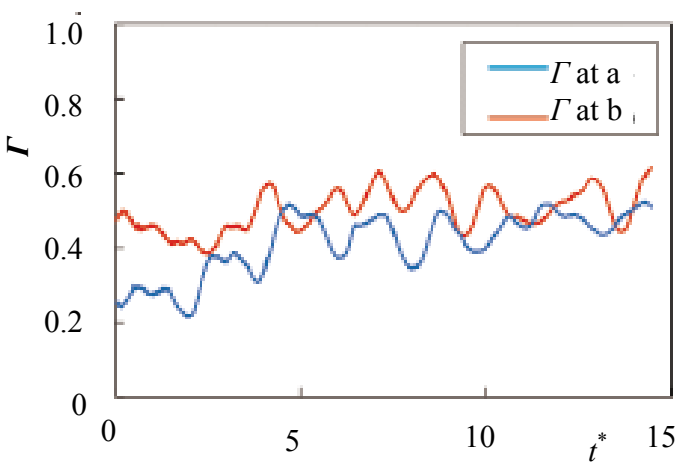

(a) $R e=95($ Pattern $\mathrm{A})$

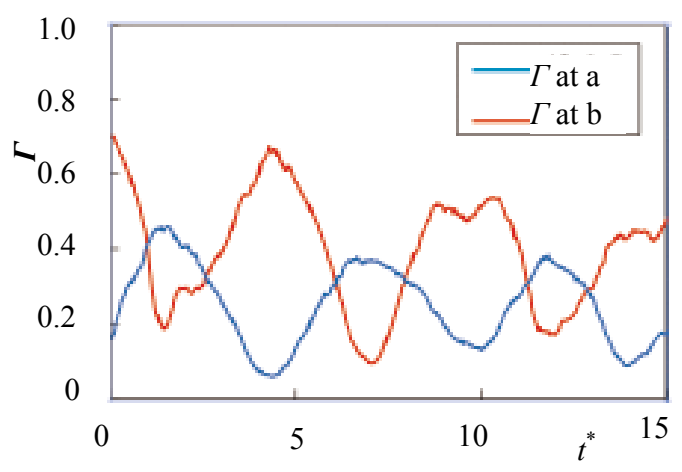

(b) $R e=476$ (Pattern B)

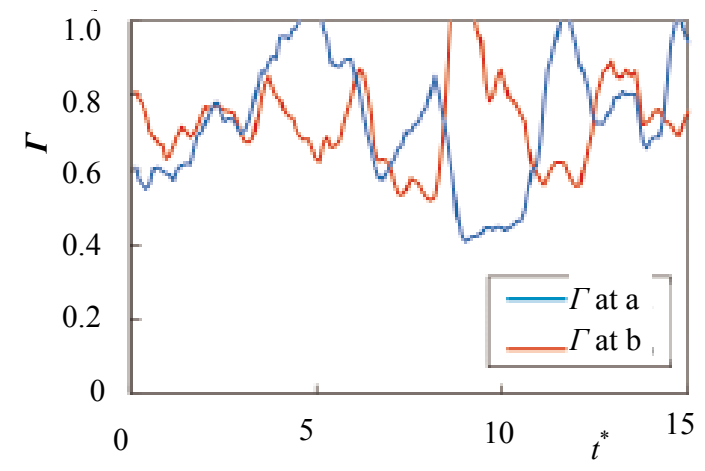

(c) $R e=2,378$ (Pattern $\mathrm{C})$

Fig. 7 Time variation of $\Gamma$ at $a$ and $b\left(C_{0}=0.02, z_{1}=40 \mathrm{~mm}\right.$, $\left.z_{2}=70 \mathrm{~mm}\right)$.

Fig. 8 shows the effect of $C_{0}$ on the vertical change in $\Gamma$, where $R e=476$ and $t^{*}=14$. The jet behavior corresponds to Pattern B. Irrespective of $C_{0}, \Gamma$ is very high near the jet centerline $(x / d=1)$, with the maximum value around the density interface $(z / d=5)$. The height at which the maximum value occurs lowers with increasing $C_{0}$ when $0.01<C_{0}<0.03$. This is because the jet density is larger. Therefore the maximum height of the jet lowers owing to the larger gravitational effect. The previous study of Shakouchi et al. [7] reports such 


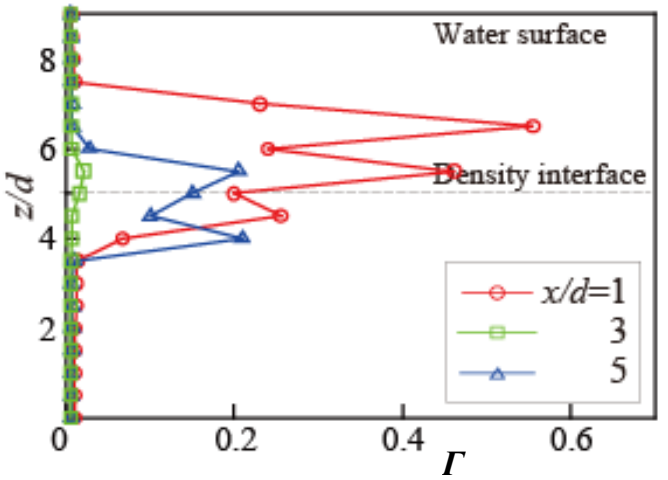

(a) $C_{0}=0.01$

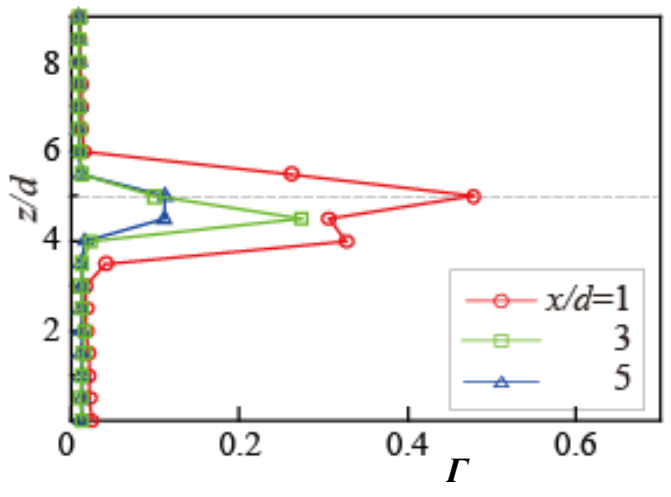

(b) $C_{0}=0.02$

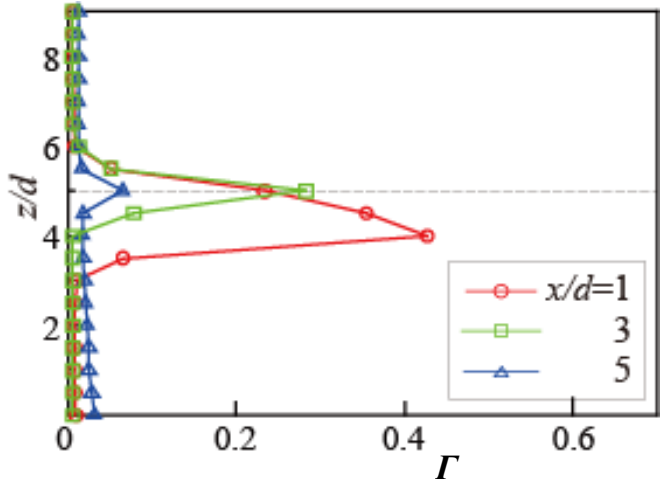

(c) $C_{0}=0.03$

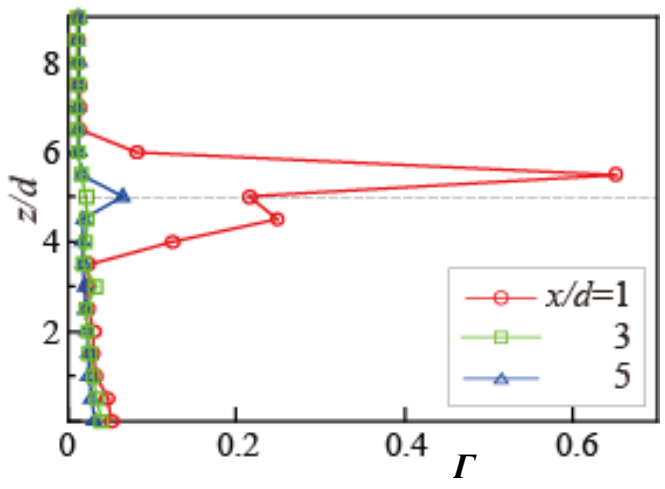

(d) $C_{0}=0.04$

Fig. 8 Effect of $C_{0}$ on the vertical distribution of $\Gamma$ at $t^{*}=14\left(R e=476, z_{1}=40 \mathrm{~mm}, z_{2}=70 \mathrm{~mm}\right)$.

effect of $C_{0}$ on the jet height. It also clarifies that the jet height can be predicted by the Froude number defined with jet velocity and the density difference between the upper and lower fluids. Note that the $\Gamma$ value at the location of $x / d=5$ far from the jet centerline lessens markedly with increasing $C_{0}$. The spread of the jet in the horizontal direction reduces, indicating the decay of the mixing.

\subsection{Distribution of Fluid Velocity}

Fig. 9 shows the velocity distribution in the vertical cross-section ( $\mathrm{x}-\mathrm{z}$ plane) passing through the jet centerline. The measured conditions are the same as those for the flow visualization depicted in Fig. 3. The time-averaged velocity $u$, calculated in a short time period of $13.75 \leq t^{*} \leq 14.25$, is shown. The color map indicates the distribution of $|u| / U_{0}$. Fig. 9a shows the distribution at $R e=95$. The jet loses its vertically upward velocity at the top, and the horizontal and downward flows occur there. When $R e=476$, the jet penetrates the density interface but loses its vertically upward velocity just after the penetration, as shown in Fig. 9b. Since $|u| / U_{0}$ is relatively higher along the interface, the intrusion is reconfirmed. The distribution at $R e=2,378$ is shown in Fig. 9c. The jet reaches the water surface, and the jet width spreads greatly in the horizontal direction in the upper fluid. One can observe the active mixing between the jet and the ambient fluid.

The vertical component of the velocity $u$ shown in Fig. 9, $u_{z}$, distributes as plotted in Fig. 10. The distribution on the density interface at $z / d=5$ and those on the upper and lower horizontal sections at $z / d=7$ and 3 respectively are depicted. Fig. 10a shows the distribution at $R e=95$. The velocity $u_{z}$ on the lower section $(z / d=3)$ takes its maximum value at the jet centerline $(x / d=0)$. The maximum value also appears at the jet centerline on the density interface $(z / d=5)$. But it is extremely lower. The velocity is not observed 


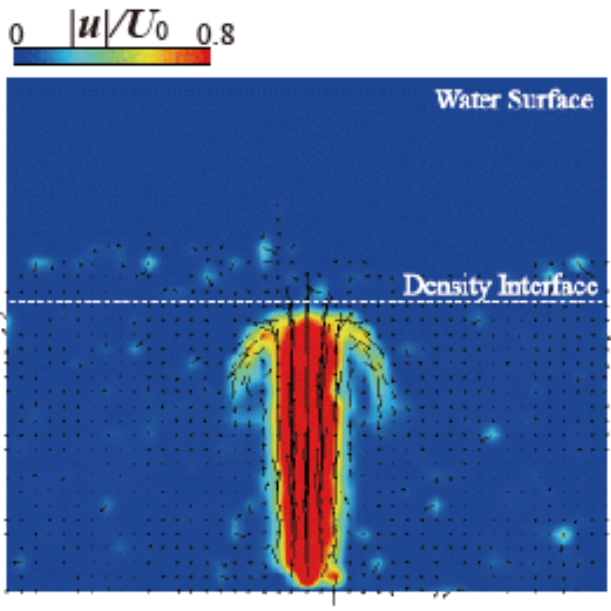

(a) $R e=95$ (Pattern A)

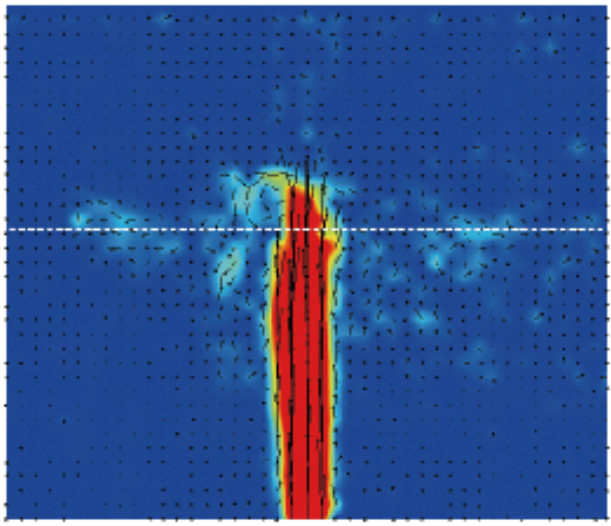

(b) $R e=476$ (Pattern B)

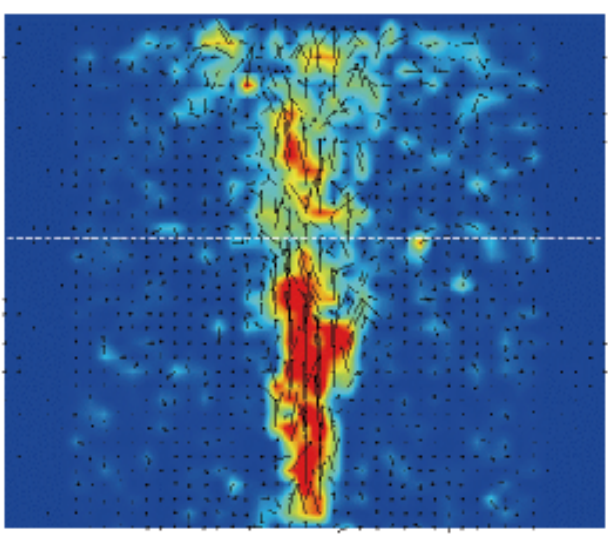

(c) $R e=2,378($ Pattern $\mathrm{C})$

Fig. 9 Velocity distribution in the central vertical cross-section at $t^{*}=14\left(C_{0}=0.02, z_{1}=40 \mathrm{~mm}, z_{2}=70 \mathrm{~mm}\right)$.

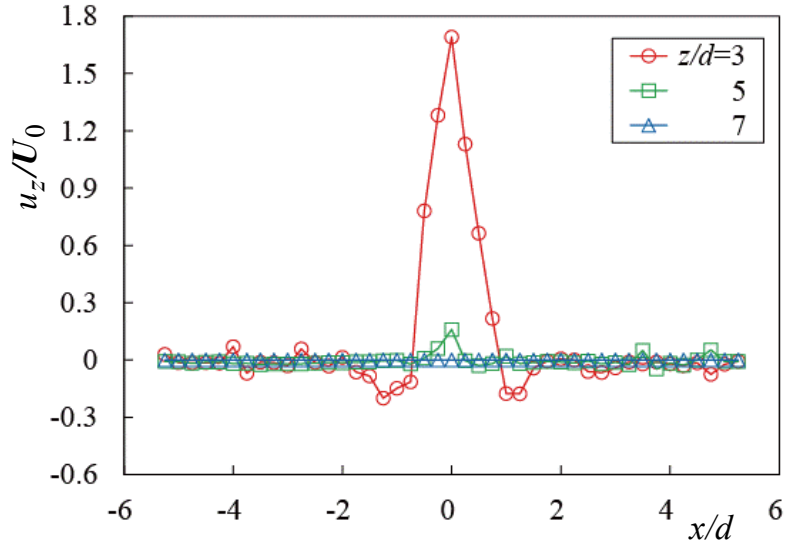

(a) $R e=95$ (Pattern A)

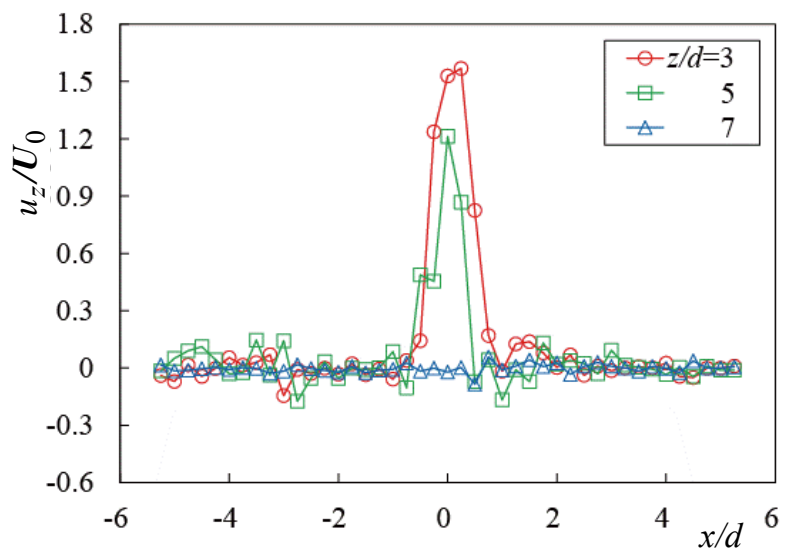

(b) $R e=476$ (Pattern $\mathrm{B})$

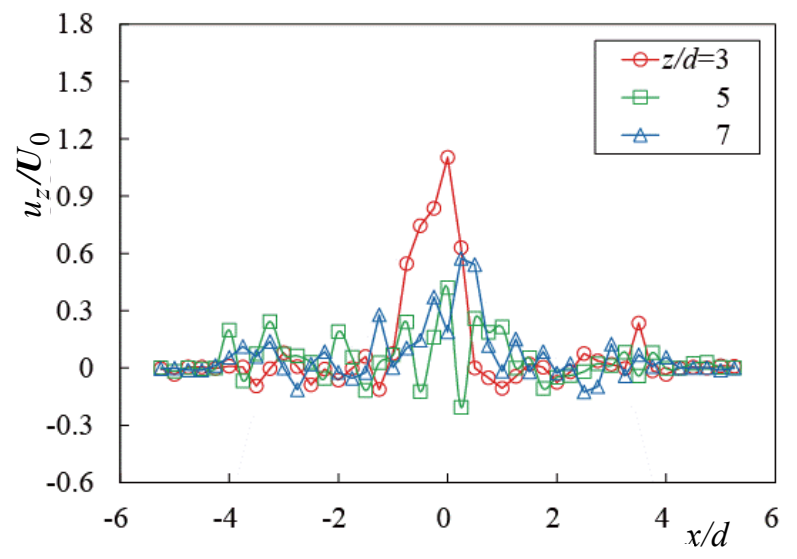

(c) $R e=2,378($ Pattern C)

Fig. 10 Lateral distribution of the vertical velocity at $t^{*}=$ $14\left(C_{0}=0.02, z_{1}=40 \mathrm{~mm}, z_{2}=70 \mathrm{~mm}\right)$. 


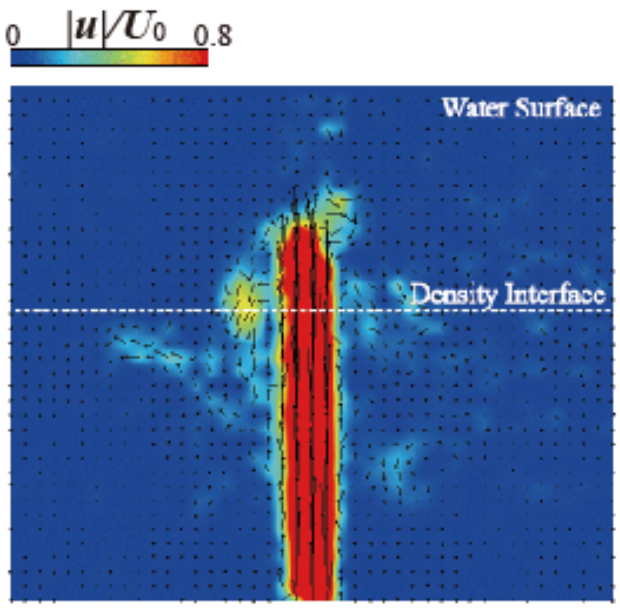

(a) $C_{0}=0.01$

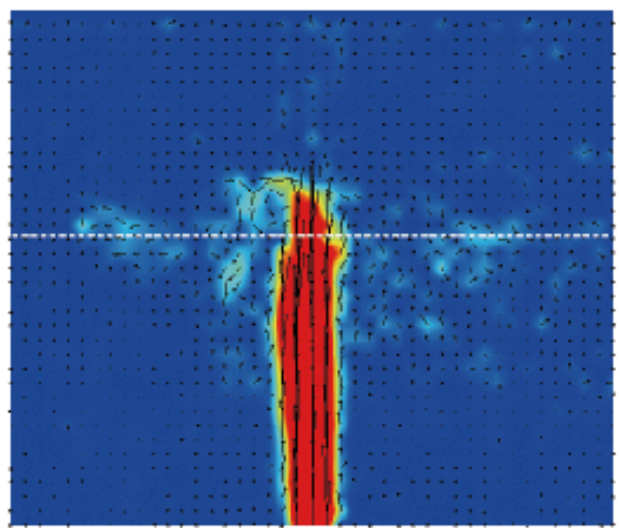

(b) $C_{0}=0.02$

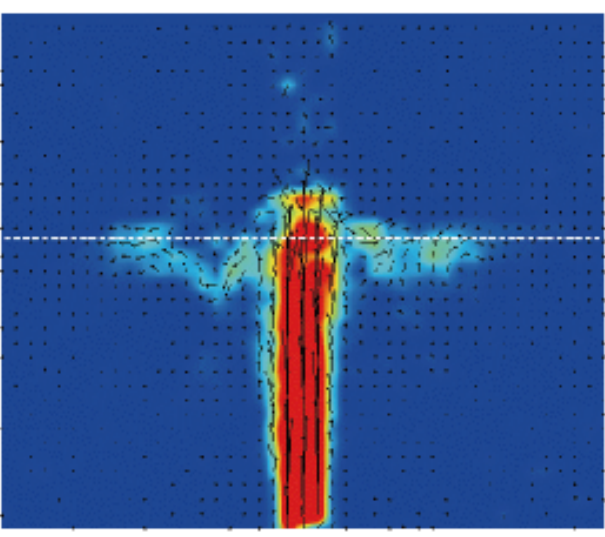

(c) $C_{0}=0.03$

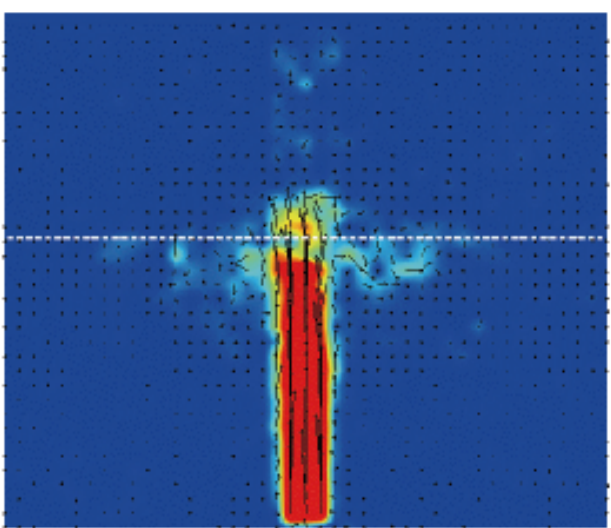

(d) $C_{0}=0.04$

Fig. 11 Effect of $C_{0}$ on the velocity distribution in the central vertical cross-section at $t^{*}=14\left(\operatorname{Re}=476, z_{1}=40 \mathrm{~mm}, z_{2}=70\right.$ mm).

in the region far from the jet centerline. There is no flow on the upper section $(z / d=7)$. The velocity $u_{\mathrm{z}}$ on the lower section $(z / d=3)$ reaches the minimum value at $x / d=-1$ and 1 on the right and the left sides of the jet centerline. This is the result of the downward flow from the top of the jet, as shown in Figs. 3 and 9. When $R e=$ 476, such downward flow does not appears on the lower section $(z / d=3)$, as found from Fig. $10 \mathrm{~b}$. Comparing the velocity on the interface $(z / d=5)$ with the result at $R e=95$, the maximum value is rather larger and the velocity $u_{z}$ also occurs in the region far from the jet centerline. One can reconfirm the active mixing along the density interface. But the flow hardly exists on the upper section $(z / d=7)$, just as in the case of $R e=95$ Fig. 10c shows the distribution at $R e=$ 2,378 . The maximum velocity on the density interface lowers and the momentum of the jet markedly diffuses in the horizontal direction. The velocity is induced even in the upper fluid. The mixing is highly activated.

Fig. 11 shows the effect of $C_{0}$ on the velocity distribution in the vertical cross-section passing through the jet centerline, where $R e=476, z_{1}=40 \mathrm{~mm}$ and $z_{2}=70 \mathrm{~mm}$. The time-averaged velocity $u$ at 13.75 $\leq t^{*} \leq 14.25$ is plotted. The jet behavior corresponds to Pattern B. When $C_{0}=0.01$, the jet loses its vertically upward velocity after penetrating the density interface. The height, at which the jet loses the velocity, or the maximum height of the jet lowers with increasing $C_{0}$. 


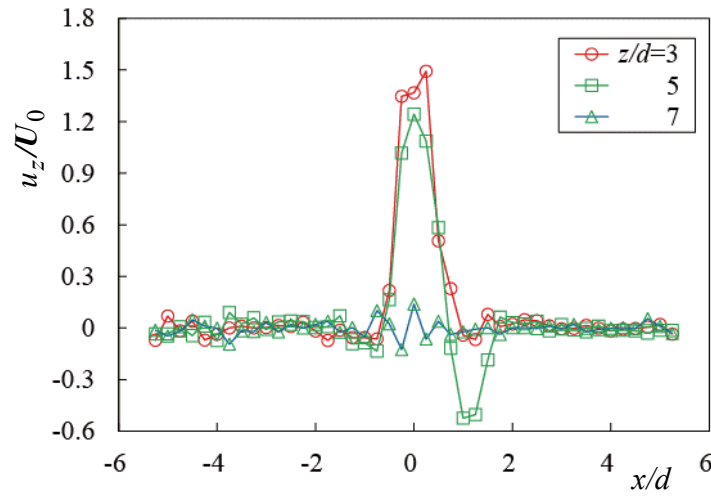

(a) $C_{0}=0.01$

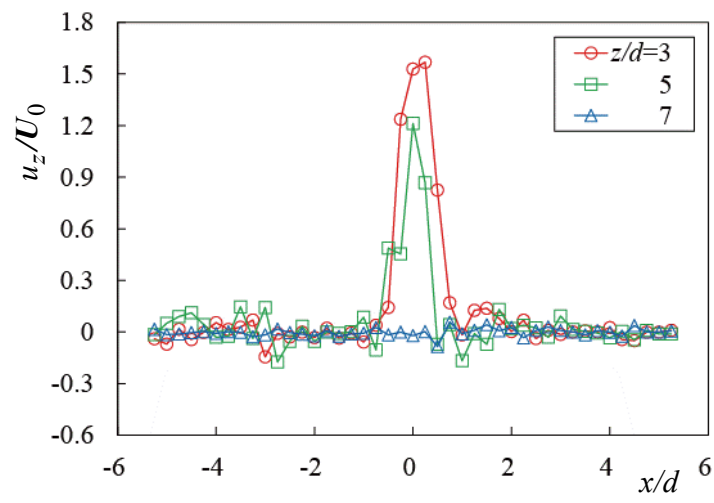

(b) $C_{0}=0.02$

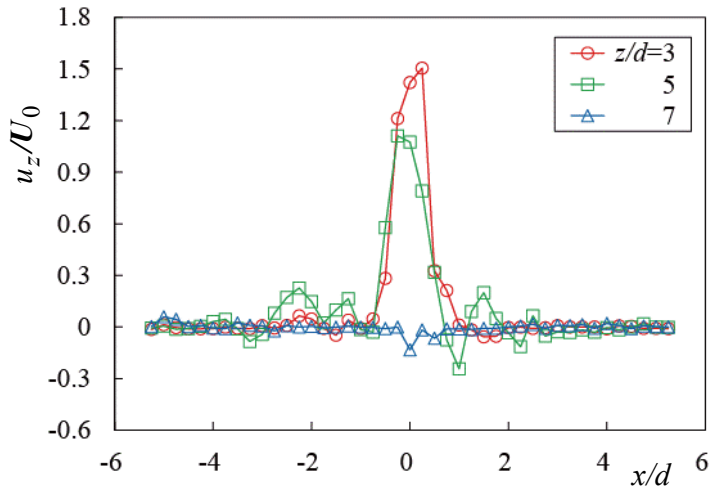

(c) $C_{0}=0.03$

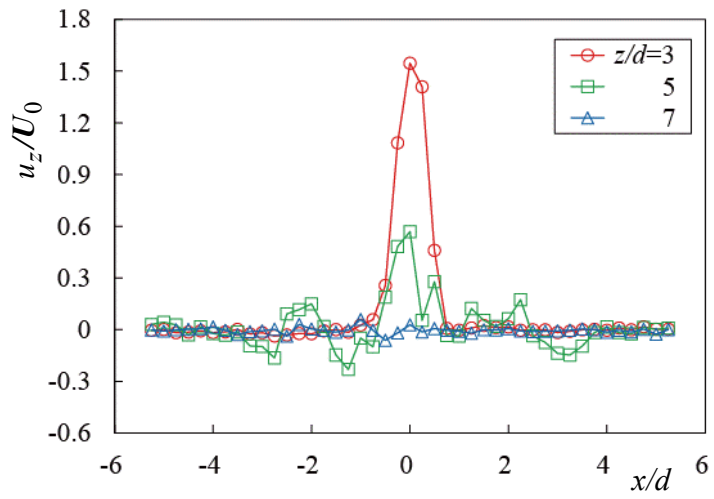

(d) $C_{0}=0.04$

Fig. 12 Effect of $C_{0}$ on the lateral distribution of vertical velocity at $t^{*}=14\left(\operatorname{Re}=476, z_{1}=40 \mathrm{~mm}, z_{2}=70 \mathrm{~mm}\right)$.

This is because the jet density is heightened, and therefore the gravitational effect appears more in the upper fluid. When $C_{0}=0.03$ and 0.04 , the fluid descending from the top of the jet flows along the density interface with a heaving motion.

The vertical component of the velocity shown in Fig. $11, u_{z}$, is plotted in Fig. 12. The velocity on the lower section $(z / d=3)$ is less affected by $C_{0}$. But the maximum value on the density interface $(z / d=5)$ lowers with increasing $C_{0}$. When $C_{0}=0.03$ and 0.04 , the absolute value of $u_{z}$ is relatively larger at the periphery of the jet $(1 \leq|x| / d \leq 3)$ on the density interface. This is because the top of the jet is located near the density interface, and accordingly the heaving flow exists along the interface as shown in Fig. 11.

\section{Conclusions}

The mixing phenomena of a two-layer density-stratified fluid induced by a jet in a rectangular tank are investigated. The upper and lower fluids are water and a $\mathrm{NaCl}$-water solution, respectively, with the lower fluid issued vertically upward from a nozzle on the tank bottom. The flow visualization is conducted, and the measurement of the concentration distribution of the water paint issued with the jet is performed. The distribution of the fluid velocity is also measured. The effects of the jet Reynolds number $R e$ and the mass concentration of the $\mathrm{NaCl}$-solution water $C_{0}$ are investigated. The results are summarized as follows:

(1) The jet behavior relative to the density interface is classified into three patterns $\mathrm{A}, \mathrm{B}$ and $\mathrm{C}$ according to $R e$ and $C_{0}$. The vertical distribution of $\Gamma$ is also specific to the jet behavior;

(2) In Pattern A, maximum $\Gamma$ occurs slightly below the density interface. It lowers with increasing distance from the jet centerline. In Pattern $\mathrm{B}, \Gamma$ reaches the maximum on the interface. The maximum value is larger than that in Pattern A. In Pattern C, $\Gamma$ is high in 
the broader region above the density interface;

(3) In Pattern B, $\Gamma$ takes the maximum value at the height near the density interface irrespective of $C_{0}$. The height lowers with increasing $C_{0}$;

(4) The fluid velocity also depends on the jet behavior relative to the density interface. In Pattern A, the jet loses its vertically upward velocity when reaching the interface, and the horizontal and downward flows occur there. In Pattern B, the jet loses the vertically upward velocity just after the penetration of the density interface and the fluid velocity occurs along the interface. In Pattern $C$, the jet velocity diffuses markedly in the horizontal direction in the upper fluid;

(5) In Pattern $C$, the height at which the jet loses the velocity lowers with increasing $C_{0}$. When $C_{0}=0.03$ and 0.04 , the absolute value of the vertical velocity is relatively larger at the periphery of the jet along the density interface.

\section{References}

[1] Baines, W. D., Turner, J. S., and Campbell, I. H. 1990. "Turbulent Fountains in an Open Chamber." Journal of Fluid Mechanics 212 (Mar.): 557-92.
[2] Bloomfield, L. J., and Kerr, R. C. 1998. "Turbulent Fountains in a Stratified Fluid." Journal of Fluid Mechanics 358 (Mar.): 335-56.

[3] Bloomfield, L. J., and Kerr, R. C., 1999. "Turbulent Fountains in a Confined Stratified Environment." Journal of Fluid Mechanics 389 (June): 27-54.

[4] Friedman, P., and Katz, J. 2000. "Rise Height for Negatively Buoyant Fountains and Depth of Penetration for Negatively Buoyant Jets Impinging an Interface." Journal of Fluids Engineering 122 (Apr.): 779-82.

[5] Lin, Y. J. P., and Linden, P. F. 2005. "The Entrainment due to a Turbulent Fountain at a Density Interface." Journal of Fluid Mechanics 542 (Nov.): 25-52.

[6] Ansong, J. K., Kyba, P. J, and Sutherland, B. R. 2008. "Fountains Impinging upon a Density Interface." Journal of Fluid Mechanics 595 (Jan.): 115-39.

[7] Shakouchi, S., Fukue, S., and Uchiyama, T. 2015. "Investigation of the Behavior of a Jet Issued into Two-Layer Density-Stratified Fluid." Journal of Flow Control, Measurement and Visualization 3 (1): 1-9.

[8] Shakouchi, S., Shimada, S., and Uchiyama, T. 2014. "Numerical Simulation of Jet Flow Issued into Density-Stratified Fluid by Vortex in Cell Method." Advances and Applications in Fluid Mechanics (accepted and in press).

[9] Simpson, J. E. 1982. "Gravity Currents in the Laboratory, Atmosphere, and Ocean." Annual Review of Fluid Mechanics 14 (Jan.): 213-34. 